\title{
Dynamics in a ratio-dependent Lotka-Volterra competitive-competitive-cooperative system with feedback controls and delays
}

\author{
Ahmadjan Muhammadhaji1* (D), Azhar Halik and Hong-Li Li'
}

${ }^{*}$ Correspondence: ahmatjanam@163.com ${ }^{1}$ College of Mathematics and System Sciences, Xinjiang University, Shengli Road, Urumqi, 830046, P.R. China

\section{Springer}

\begin{abstract}
This study investigates the dynamical behavior of a ratio-dependent Lotka-Volterra competitive-competitive-cooperative system with feedback controls and delays. Compared with previous studies, both ratio-dependent functional responses and time delays are considered. By employing the comparison method, the Lyapunov function method, and useful inequality techniques, some sufficient conditions on the permanence, periodic solution, and global attractivity for the considered system are derived. Finally, a numerical example is also presented to validate the practicability and feasibility of our proposed results.
\end{abstract}

Keywords: Ratio-dependent competitive-competitive-cooperative system; Time delay; Permanence; Feedback control; Global attractivity

\section{Introduction}

As is well known, competition and mutualism(cooperation) are two important interactions among species. Competition occurs when two species use the same resources or harm each other when seeking resources, whereas mutualism is defined as the living of two species in close association with one another with the benefit of both [1]. Notably, the Lotka-Volterra models were proposed by Lotka [2] and Volterra [3] for the first time, and now they have become the most important means to explain this type of ecological phenomenon. In particular, the Lotka-Volterra competitive model, mutualism (cooperative) model, and predator-prey model characterize competitive, cooperative, and predator-prey interactions between species that are of great interest in the study of dynamical behaviors of systems [4-17]. However, pure competition as described by the Lotka-Volterra model often results in species exclusion or coexistence with reduced carrying capacity of both species and does not help the coexistence of multiple species, although it is a driving force for natural selection [1]. Hence, when modeling we should consider more interactions between species such as competition, cooperation, and predator-prey [18-26].

(c) The Author(s) 2021. This article is licensed under a Creative Commons Attribution 4.0 International License, which permits use, sharing, adaptation, distribution and reproduction in any medium or format, as long as you give appropriate credit to the original author(s) and the source, provide a link to the Creative Commons licence, and indicate if changes were made. The images or other third party material in this article are included in the article's Creative Commons licence, unless indicated otherwise in a credit line to the material. If material is not included in the article's Creative Commons licence and your intended use is not permitted by statutory regulation or exceeds the permitted use, you will need to obtain permission directly from the copyright holder. To view a copy of this licence, visit http://creativecommons.org/licenses/by/4.0/. 
For example, in [18], the authors considered the following delayed competitivecooperative systems:

$$
\begin{aligned}
\dot{z}_{1}(t)= & z_{1}(t)\left(r_{1}(t)-a_{11}^{1}(t) z_{1}(t-\tau)-a_{11}^{2}(t) z_{1}(t-2 \tau)\right. \\
& \left.-a_{12}(t) z_{2}(t-2 \tau)+a_{13}(t) z_{3}(t-\tau)\right), \\
\dot{z}_{2}(t)= & z_{2}(t)\left(r_{2}(t)-a_{21}(t) z_{1}(t-2 \tau)-a_{22}^{1}(t) z_{2}(t-\tau)\right. \\
& \left.-a_{22}^{2}(t) z_{2}(t-2 \tau)+a_{23}(t) z_{3}(t-\tau)\right), \\
\dot{z}_{3}(t)= & z_{3}(t)\left(r_{3}(t)+a_{31}(t) z_{1}(t-\tau)+a_{32}(t) z_{2}(t-\tau)\right. \\
& \left.-a_{33}^{1}(t) z_{3}(t)-a_{33}^{2}(t) z_{3}(t-\tau)\right) .
\end{aligned}
$$

They established some sufficient conditions that ensured the system is permanent and globally attractive. In [22], the authors proposed the following Lotka-Volterra predatorprey-competition model with feedback controls:

$$
\begin{aligned}
& \dot{z}_{1}(t)=z_{1}(t)\left[\gamma_{1}(t)-b_{11}(t) z_{1}(t)-\frac{b_{12}(t) z_{2}(t)}{a_{12}(t) z_{2}(t)+z_{1}(t)}-\frac{b_{13}(t) z_{3}(t)}{a_{13}(t) z_{3}(t)+z_{1}(t)}-c_{1}(t) v_{1}(t)\right] \\
& \dot{z}_{2}(t)=z_{2}(t)\left[-\gamma_{2}(t)+\frac{b_{21}(t) z_{1}(t)}{a_{12}(t) z_{2}(t)+z_{1}(t)}-b_{23}(t) z_{3}(t)+c_{2}(t) v_{2}(t)\right] \\
& \dot{z}_{3}(t)=z_{3}(t)\left[-\gamma_{3}(t)+\frac{b_{31}(t) z_{1}(t)}{a_{13}(t) z_{3}(t)+z_{1}(t)}-b_{32}(t) z_{2}(t)+c_{3}(t) v_{3}(t)\right] \\
& \dot{v}_{1}(t)=q_{1}(t)-e_{1}(t) v_{1}(t)+f_{1}(t) z_{1}(t) \\
& \dot{v}_{2}(t)=q_{2}(t)-e_{2}(t) v_{2}(t)-f_{2}(t) z_{2}(t) \\
& \dot{v}_{3}(t)=q_{3}(t)-e_{3}(t) v_{3}(t)-f_{3}(t) z_{3}(t)
\end{aligned}
$$

They have obtained some sufficient conditions for the permanence, global attractivity, existence, and stability of the positive periodic solution for system (1.2) by using a comparison theorem, constructing a suitable Lyapunov function, the fixed-point theory, and a new analysis method. In [22] the authors presented an open problem adding a delay term to the proposed model (1.2) and studying the dynamical properties of system (1.2). In [25], the authors further analyzed systems (1.1) and (1.2) and subsequently proposed the following delayed Lotka-Volterra competitive-competitive-cooperative model with feedback controls:

$$
\begin{aligned}
\dot{z}_{1}(t)= & z_{1}(t)\left[r_{1}(t)-a_{11}^{1}(t) z_{1}(t-\tau)-a_{11}^{2}(t) z_{1}(t-2 \tau)\right. \\
& \left.-a_{12}(t) z_{2}(t-2 \tau)+a_{13}(t) z_{3}(t-\tau)-c_{1}(t) v_{1}(t)\right], \\
\dot{z}_{2}(t)= & z_{2}(t)\left[r_{2}(t)-a_{21}(t) z_{1}(t-2 \tau)-a_{22}^{1}(t) z_{2}(t-\tau)\right. \\
& \left.-a_{22}^{2}(t) z_{2}(t-2 \tau)+a_{23}(t) z_{3}(t-\tau)+c_{2}(t) v_{2}(t)\right], \\
\dot{z}_{3}(t)= & z_{3}(t)\left[r_{3}(t)+a_{31}(t) z_{1}(t-\tau)+a_{32}(t) z_{2}(t-\tau)\right. \\
& \left.-a_{33}^{1}(t) z_{3}(t)-a_{33}^{2}(t) z_{3}(t-\tau)+c_{3}(t) v_{3}(t)\right], \\
\dot{v}_{1}(t)= & q_{1}(t)-e_{1}(t) v_{1}(t)+f_{1}(t) x_{1}(t),
\end{aligned}
$$




$$
\begin{aligned}
& \dot{v}_{2}(t)=q_{2}(t)-e_{2}(t) v_{2}(t)-f_{2}(t) x_{2}(t), \\
& \dot{v}_{3}(t)=q_{3}(t)-e_{3}(t) \nu_{3}(t)-f_{3}(t) x_{3}(t) .
\end{aligned}
$$

They further obtained sufficient conditions for the permanence and global attractivity for system (1.3) by developing a new analysis technique and constructing a new and suitable Lyapunov function.

However, to the best of our knowledge, no study has been conducted to date for dynamics on the three-species ratio-dependent Lotka-Volterra competitive-competitivecooperative system with feedback controls and delays. Therefore, based on the above models, analysis, and reasons, in this study we extend systems (1.2) and (1.3) to the following system:

$$
\begin{aligned}
\dot{x}_{1}(t)= & x_{1}(t)\left[r_{1}(t)-a_{1}(t) x_{1}(t)-\frac{b_{1}(t) x_{2}\left(t-\tau_{1}\right)}{c_{1}(t) x_{2}\left(t-\tau_{1}\right)+x_{1}\left(t-\tau_{1}\right)}\right. \\
& \left.+\frac{d_{1}(t) x_{3}\left(t-\tau_{1}\right)}{e_{1}(t) x_{3}\left(t-\tau_{1}\right)+x_{1}\left(t-\tau_{1}\right)}-f_{1}(t) u_{1}(t)\right], \\
\dot{x}_{2}(t)= & x_{2}(t)\left[r_{2}(t)-a_{2}(t) x_{2}(t)-\frac{b_{2}(t) x_{1}\left(t-\tau_{2}\right)}{c_{2}(t) x_{1}\left(t-\tau_{2}\right)+x_{2}\left(t-\tau_{2}\right)}\right. \\
& \left.+\frac{d_{2}(t) x_{3}\left(t-\tau_{2}\right)}{e_{2}(t) x_{3}\left(t-\tau_{2}\right)+x_{2}\left(t-\tau_{2}\right)}+f_{2}(t) u_{2}(t)\right], \\
\dot{x}_{3}(t)= & x_{3}(t)\left[r_{3}(t)-a_{3}(t) x_{3}(t)+\frac{g_{1}(t) x_{1}\left(t-\tau_{3}\right)}{e_{1}(t) x_{3}\left(t-\tau_{3}\right)+x_{1}\left(t-\tau_{3}\right)}\right. \\
& \left.+\frac{g_{2}(t) x_{2}\left(t-\tau_{3}\right)}{e_{2}(t) x_{3}\left(t-\tau_{3}\right)+x_{2}\left(t-\tau_{3}\right)}+f_{3}(t) u_{3}(t)\right], \\
\dot{u}_{1}(t)= & q_{1}(t)-p_{1}(t) u_{1}(t)+h_{1}(t) x_{1}(t), \\
\dot{u}_{2}(t)= & q_{2}(t)-p_{2}(t) u_{2}(t)-h_{2}(t) x_{2}(t), \\
\dot{u}_{3}(t)= & q_{3}(t)-p_{3}(t) u_{3}(t)-h_{3}(t) x_{3}(t) .
\end{aligned}
$$

The aim of this study is to use the inequality techniques, comparison method and to construct suitable Lyapunov functionals to establish some new and sufficient conditions on the permanence, periodic solution, and global attractivity for system (1.4).

\section{Preliminaries}

In system (1.4), $x_{i}(t)(i=1,2,3)$ represents the density of three species $x_{i}(i=1,2,3)$ at time t. $r_{i}(t)(i=1,2,3)$ represents the intrinsic growth rate of three species $x_{i}(i=1,2,3)$ at time t. $a_{i}(t)(i=1,2,3)$ represents the intrapatch restriction density of three species $x_{i}(i=1,2,3)$ at time $t . d_{i}(t), g_{i}(t)(i=1,2)$ represent the cooperative coefficients between species $x_{1}, x_{2}$, and $x_{3}$ at time $t . b_{i}(t)(i=1,2)$ represents the competitive coefficients between species $x_{1}$ and $x_{2}$ at time $t . u_{i}(t)(i=1,2,3)$ represents the indirect control variables at time $t$. $q_{i}(t), p_{i}(t), h_{i}(t), f_{i}(t)(i=1,2,3)$ are the feedback control coefficients at time $t . \tau_{i}(i=1,2,3)$ is a positive constant. 
In this paper, the initial conditions for system (1.4) take the following form:

$$
\begin{aligned}
& x_{i}(t)=\varphi_{i}(t) \quad \text { for all } t \in[-\tau, 0], i=1,2,3, \\
& u_{i}(t)=\phi_{i}(t) \quad \text { for all } t \in[0,+\infty), i=1,2,3,
\end{aligned}
$$

where $\varphi_{i}(0)>0, \phi_{i}(0)>0(i=1,2,3)$, and $\tau=\max \left\{\tau_{i}(i=1,2,3)\right\}$.

For system (1.4) we introduce the following assumptions:

$\left(H_{1}\right) r_{i}(t), a_{i}(t) q_{i}(t), p_{i}(t), h_{i}(t), f_{i}(t)(i=1,2,3)$ and $b_{i}(t), c_{i}(t), d_{i}(t), e_{i}(t), g_{i}(t)(i=1,2)$ are continuous, bounded, and positive functions on $[0,+\infty)$.

$\left(H_{2}\right) r_{i}(t), a_{i}(t) q_{i}(t), p_{i}(t), h_{i}(t), f_{i}(t)(i=1,2,3)$ and $b_{i}(t), c_{i}(t), d_{i}(t), e_{i}(t), g_{i}(t)(i=1,2)$ are all continuously positive $\omega$-periodic functions on $[0, \omega]$.

For a continuous and bounded function $f(t)$ defined on $[0,+\infty)$, we define $f^{L}=$ $\inf _{t \in[0,+\infty)}\{f(t)\}$ and $f^{M}=\sup _{t \in[0,+\infty)}\{f(t)\}$.

In this paper, we need the following definition and lemmas.

Definition 2.1 ([17]) System (1.4) is called permanent if there exist positive constants $M_{i}, N_{i}, m_{i}, n_{i}(i=1,2,3)$ and $T>0$ such that $m_{i} \leq x_{i}(t) \leq M_{i}, n_{i} \leq u_{i}(t) \leq N_{i}(i=1,2,3)$ for any positive solution $Z(t)=\left(x_{1}(t), x_{2}(t), x_{3}(t), u_{1}(t), u_{2}(t), u_{3}(t)\right)$ of system (1.4) with the initial values (2.1) as $t>T$.

Lemma 2.1 ([6]) Consider the following equation:

$$
\dot{u}(t)=u(t)\left(d_{1}-d_{2} u(t)\right)
$$

where $d_{2}>0$, we have

(1) If $d_{1}>0$, then $\lim _{t \rightarrow+\infty} u(t)=d_{1} / d_{2}$.

(2) If $d_{1}<0$, then $\lim _{t \rightarrow+\infty} u(t)=0$.

Lemma 2.2 ([25]) If $a>0, b>0$, and $\dot{x}(t) \geq(\leq) b-a x(t)$, when $t \geq 0$ and $x(0)>0$, we have

$$
x(t) \geq(\leq) \frac{b}{a}\left[1+\left(\frac{a x(0)}{b}-1\right) e^{-a t}\right]
$$

Consider the following periodic differential equation with solution $x(t, 0, \Phi)$ :

$$
\frac{d x}{d t}=F\left(t, x_{t}\right)
$$

where $F\left(t, x_{t}\right)$ is an $n$-dimensional continuous functional and $x(t) \in R^{n}, x(t, 0, \Phi)=$ $\left(x_{1}(t, 0, \Phi), x_{2}(t, 0, \Phi), \ldots, x_{n}(t, 0, \Phi)\right)$ is a solution of the functional differential equation with the initial condition $x_{0}=\Phi$.

Lemma 2.3 ([26]) If there exist positive constants $m$ and $M$ for any $\Phi \in C_{+}^{n}[-\tau, 0]$ such that

$$
m<\liminf _{t \rightarrow \infty} x_{i}(t, 0, \Phi) \leq \limsup _{t \rightarrow \infty} x_{i}(t, 0, \Phi)<M, \quad i=1,2, \ldots, n,
$$

then system (2.2) admits at least one positive $\omega$-periodic solution. 


\section{Permanence and periodic solution}

In this section, we obtain some new and sufficient conditions for the permanence and periodic solution of system (1.4).

Theorem 3.1 Let $x(t)=\left(x_{1}(t), x_{2}(t), x_{3}(t), u_{1}(t), u_{2}(t), u_{3}(t)\right)$ be any positive solution of system (1.4) with the initial conditions (2.1). Assume that $\left(H_{1}\right)$ holds, then there exist positive constants $M_{1}, M_{2}, M_{3}, N_{1}, N_{2}, N_{3}, T$ and $m_{3}, n_{1}$ such that $x_{i}(t) \leq M_{i}(i=1,2,3), u_{i}(t) \leq$ $N_{i}(i=1,2,3)$ and $x_{3}(t) \geq m_{3}, u_{1}(t) \geq n_{1}$ as $t>T$.

Proof From the first equation of system (1.4), for $t \geq \tau$, we have

$$
\dot{x}_{1}(t) \leq x_{1}(t)\left[r_{1}^{M}+\frac{d_{1}^{M}}{e_{1}^{L}}-a_{1}^{L} x_{1}(t)\right] .
$$

Then, by Lemma 2.1 and the comparison theorem, there exists a constant $T_{0}>0$ such that

$$
x_{1}(t) \leq \frac{r_{1}^{M}+\frac{d_{1}^{M}}{e_{1}^{L}}}{a_{1}^{L}}=M_{1} \quad \text { as } t>T_{0} .
$$

By the fourth equation of system (1.4), for $t>T_{0}$, we have

$$
\dot{u}_{1}(t) \leq q_{1}^{M}+h_{1}^{M} M_{1}-p_{1}^{L} u_{1}(t) .
$$

Then, by Lemma 2.2 and the comparison theorem, there exists a constant $T_{1}>0$ such that

$$
u_{1}(t) \leq \frac{q_{1}^{M}+h_{1}^{M} M_{1}}{p_{1}^{L}}=N_{1} \quad \text { as } t>T_{1} .
$$

From the fifth and sixth equation of system (1.4), for $t \geq \tau$ and $i=2,3$, we have

$$
\dot{u}_{i}(t) \leq q_{i}^{M}-p_{i}^{L} u_{i}(t)
$$

Then, by Lemma 2.2 and the comparison theorem, there exists a constant $T_{2}>0$ such that

$$
u_{i}(t) \leq \frac{q_{i}^{M}}{p_{i}^{L}}=N_{i}, \quad i=2,3, \text { as } t>T_{2}
$$

Next, from the second equation of system (1.4), for $t>T_{2}$, we have

$$
\dot{x}_{2}(t) \leq x_{2}(t)\left[r_{2}^{M}+\frac{d_{2}^{M}}{e_{2}^{L}}+f_{2}^{M} N_{2}-a_{2}^{L} x_{2}(t)\right] .
$$

Then, by Lemma 2.1 and the comparison theorem, there exists a constant $T_{3}>0$ such that

$$
x_{2}(t) \leq \frac{r_{2}^{M}+\frac{d_{2}^{M}}{e_{2}^{L}}+f_{2}^{M} N_{2}}{a_{2}^{L}}=M_{2} \quad \text { as } t>T_{3} .
$$


Similar to the above discussion, from the third equation of system (1.4), there exists a constant $T_{4}>0$ such that

$$
x_{3}(t) \leq \frac{r_{3}^{M}+g_{1}^{M}+g_{2}^{M}+f_{3}^{M} N_{3}}{a_{3}^{L}}=M_{3} \quad \text { as } t>T_{4} .
$$

On the other hand, from the third equation of system (1.4), for $t \geq \tau$, we have

$$
\dot{x}_{3}(t) \geq x_{3}(t)\left[r_{3}^{L}-a_{3}^{M} x_{3}(t)\right]
$$

Then, by Lemma 2.1 and the comparison theorem, there exists a constant $T_{5}>0$ such that

$$
x_{3}(t) \geq \frac{r_{3}^{L}}{a_{3}^{M}}=m_{3} \quad \text { as } t>T_{5}
$$

Finally, from the fourth equation of system (1.4), for $t \geq \tau$, we have

$$
\dot{u}_{1}(t) \geq q_{1}^{L}-p_{1}^{M} u_{1}(t)
$$

Then, by Lemma 2.2 and the comparison theorem, there exists a constant $T_{6}>0$ such that

$$
u_{1}(t) \geq \frac{q_{1}^{L}}{p_{1}^{M}}=n_{1} \quad \text { as } t>T_{6} .
$$

This completes the proof of Theorem 3.1.

Theorem 3.2 Assume that $\left(H_{1}\right)$ holds and

$$
\begin{aligned}
& A_{1}=r_{1}^{L}+\frac{d_{1}^{L} m_{3}}{e_{1}^{M} M_{3}+M_{1}}-\frac{b_{1}^{M}}{c_{1}^{L}}-f_{1}^{M} N_{1}>0, \quad B_{2}=q_{2}^{L}-h_{2}^{M} M_{2}>0, \\
& A_{2}=r_{2}^{L}+\frac{d_{2}^{L} m_{3}}{e_{2}^{M} M_{3}+M_{2}}-\frac{b_{2}^{M}}{c_{2}^{L}}>0, \quad B_{3}=q_{3}^{L}-h_{3}^{M} M_{3}>0,
\end{aligned}
$$

then system (1.4) is permanent, where $M_{1}, M_{2}, M_{3}, N_{1}$, and $m_{3}$ are defined in Theorem 3.1.

Proof Firstly, from the first and second equation of system (1.4), we can obtain a sufficiently large positive constant $T_{1}^{M}$ such that

$$
\dot{x}_{i}(t) \geq x_{i}(t)\left[A_{i}-a_{i}^{M} x_{i}(t)\right] \quad \text { as } t>T_{1}^{M}, i=1,2 .
$$

Then, by Lemma 2.2 and the comparison theorem, there exists a constant $T_{2}^{M}>T_{1}^{M}$ such that

$$
x_{i}(t) \geq \frac{A_{i}}{a_{i}^{M}}=m_{i} \quad \text { as } t>T_{2}^{M}, i=1,2 .
$$

Next, from the fifth and sixth equation of system (1.4), we can obtain a sufficiently large positive constant $T_{1}^{N}$ such that

$$
\dot{u}_{i}(t) \geq B_{i}-p_{i}^{M} u_{i}(t) \quad \text { as } t>T_{1}^{N}, i=2,3 .
$$


Then, by Lemma 2.2 and the comparison theorem, there exists a constant $T_{2}^{N}>T_{1}^{N}$ such that

$$
u_{i}(t) \geq \frac{B_{i}}{p_{i}^{M}}=n_{i} \quad \text { as } t>T_{2}^{N}, i=2,3
$$

This completes the proof of Theorem 3.2.

As a direct result of Lemma 2.3, from Theorem 3.1 and Theorem 3.2 we have the following.

Corollary 3.1 Assume that $\left(H_{2}\right)$ holds and $A_{1}>0, A_{2}>0, B_{2}>0, B_{3}>0$, then system (1.4) is permanent and has at least one positive $\omega$-periodic solution.

\section{Global attractivity}

In this section, we obtain the sufficient conditions for the global attractivity of system (1.4). Firstly, for convenience we denote

$$
\begin{aligned}
& C_{1}(t)=\frac{b_{1}(t) y_{2}\left(t-\tau_{1}\right)}{\gamma_{1}(t)} \leq \frac{b_{1}^{M} M_{2}}{\gamma_{1}^{L}}=C_{1}^{M}, \quad C_{2}(t)=\frac{b_{1}(t) y_{1}\left(t-\tau_{1}\right)}{\gamma_{1}(t)} \leq \frac{b_{1}^{M} M_{1}}{\gamma_{1}^{L}}=C_{2}^{M}, \\
& C_{3}(t)=\frac{d_{1}(t) y_{3}\left(t-\tau_{1}\right)}{\gamma_{2}(t)} \leq \frac{d_{1}^{M} M_{3}}{\gamma_{2}^{L}}=C_{3}^{M}, \quad C_{4}(t)=\frac{d_{1}(t) y_{1}\left(t-\tau_{1}\right)}{\gamma_{2}(t)} \leq \frac{d_{1}^{M} M_{1}}{\gamma_{2}^{L}}=C_{4}^{M}, \\
& C_{5}(t)=\frac{b_{2}(t) y_{2}\left(t-\tau_{2}\right)}{\gamma_{3}(t)} \leq \frac{b_{2}^{M} M_{2}}{\gamma_{3}^{L}}=C_{5}^{M}, \quad C_{6}(t)=\frac{b_{2}(t) y_{1}\left(t-\tau_{2}\right)}{\gamma_{3}(t)} \leq \frac{b_{2}^{M} M_{1}}{\gamma_{3}^{L}}=C_{6}^{M}, \\
& C_{7}(t)=\frac{d_{2}(t) y_{3}\left(t-\tau_{2}\right)}{\gamma_{4}(t)} \leq \frac{d_{2}^{M} M_{3}}{\gamma_{4}^{L}}=C_{7}^{M}, \quad C_{8}(t)=\frac{d_{2}(t) y_{2}\left(t-\tau_{2}\right)}{\gamma_{4}(t)} \leq \frac{d_{2}^{M} M_{2}}{\gamma_{4}^{L}}=C_{8}^{M}, \\
& C_{9}(t)=\frac{g_{1}(t) e_{1}(t) y_{3}\left(t-\tau_{3}\right)}{\gamma_{5}(t)} \leq \frac{g_{1}^{M} e_{1}^{M} M_{3}}{\gamma_{2}^{L}}=C_{9}^{M}, \\
& C_{10}(t)=\frac{g_{1}(t) e_{1}(t) y_{1}\left(t-\tau_{3}\right)}{\gamma_{5}(t)} \leq \frac{g_{1}^{M} e_{1}^{M} M_{1}}{\gamma_{2}^{L}}=C_{10}^{M}, \\
& C_{11}(t)=\frac{g_{2}(t) e_{2}(t) y_{3}\left(t-\tau_{3}\right)}{\gamma_{6}(t)} \leq \frac{g_{2}^{M} e_{2}^{M} M_{3}}{\gamma_{4}^{L}}=C_{11}^{M}, \\
& C_{12}(t)=\frac{g_{2}(t) e_{2}(t) y_{2}\left(t-\tau_{3}\right)}{\gamma_{6}(t)} \leq \frac{g_{2}^{M} e_{2}^{M} M_{2}}{\gamma_{4}^{L}}=C_{12}^{M}, \\
& D_{1}=a_{1}^{L}-C_{1}^{M}-C_{3}^{M}-C_{5}^{M}-C_{9}^{M}-h_{1}^{M}, \quad D_{2}=a_{2}^{L}-C_{2}^{M}-C_{6}^{M}-C_{7}^{M}-C_{11}^{M}-h_{2}^{M} \text {, } \\
& D_{3}=a_{3}^{L}-C_{4}^{M}-C_{8}^{M}-C_{10}^{M}-C_{12}^{M}-h_{3}^{M}, \quad E_{i}=p_{i}^{L}-f_{i}^{M} \quad(i=1,2,3) \text {, } \\
& G=\min \left\{D_{i}, E_{i}(i=1,2,3)\right\} \text {, }
\end{aligned}
$$

where

$$
\begin{aligned}
& \gamma_{1}(t)=\left(c_{1}(t) x_{2}\left(t-\tau_{1}\right)+x_{1}\left(t-\tau_{1}\right)\right)\left(c_{1}(t) y_{2}\left(t-\tau_{1}\right)+y_{1}\left(t-\tau_{1}\right)\right), \\
& \gamma_{1}^{L}=\left(c_{1}^{L} m_{2}+m_{1}\right)^{2}, \\
& \gamma_{2}(t)=\left(e_{1}(t) x_{3}\left(t-\tau_{1}\right)+x_{1}\left(t-\tau_{1}\right)\right)\left(e_{1}(t) y_{3}\left(t-\tau_{1}\right)+y_{1}\left(t-\tau_{1}\right)\right),
\end{aligned}
$$




$$
\begin{aligned}
& \gamma_{2}^{L}=\left(e_{1}^{L} m_{3}+m_{1}\right)^{2}, \\
& \gamma_{3}(t)=\left(c_{2}(t) x_{1}\left(t-\tau_{2}\right)+x_{2}\left(t-\tau_{2}\right)\right)\left(c_{2}(t) y_{1}\left(t-\tau_{2}\right)+y_{2}\left(t-\tau_{2}\right)\right), \\
& \gamma_{3}^{L}=\left(c_{2}^{L} m_{1}+m_{2}\right)^{2}, \\
& \gamma_{4}(t)=\left(e_{2}(t) x_{3}\left(t-\tau_{2}\right)+x_{2}\left(t-\tau_{2}\right)\right)\left(e_{2}(t) y_{3}\left(t-\tau_{2}\right)+y_{2}\left(t-\tau_{2}\right)\right), \\
& \gamma_{4}^{L}=\left(e_{2}^{L} m_{3}+m_{2}\right)^{2}, \\
& \gamma_{5}(t)=\left(e_{1}(t) x_{3}\left(t-\tau_{3}\right)+x_{1}\left(t-\tau_{3}\right)\right)\left(e_{1}(t) y_{3}\left(t-\tau_{3}\right)+y_{1}\left(t-\tau_{3}\right)\right), \\
& \gamma_{5}^{L}=\gamma_{2}^{L}, \\
& \gamma_{6}(t)=\left(e_{2}(t) x_{3}\left(t-\tau_{3}\right)+x_{2}\left(t-\tau_{3}\right)\right)\left(e_{2}(t) y_{3}\left(t-\tau_{3}\right)+y_{2}\left(t-\tau_{3}\right)\right), \\
& \gamma_{6}^{L}=\gamma_{4}^{L} .
\end{aligned}
$$

Theorem 4.1 Suppose that the conditions of Theorem 3.2 hold and $G>0$. Then system (1.4) is globally attractive.

Proof Let $\left(x_{1}(t), x_{2}(t), x_{3}(t)\right)$ and $\left(y_{1}(t), y_{2}(t), y_{3}(t)\right)$ be any two positive solutions of system (1.4). Choose positive constants $M_{i}, m_{i}(i=1,2,3)$, and $T$ such that $m_{i} \leq y_{i}(t), x_{i}(t) \leq$ $M_{i}(i=1,2,3)$ for all $t \geq T$. Firstly, let

$$
V_{1}(t)=\sum_{i=1}^{3}\left|\ln x_{i}(t)-\ln y_{i}(t)\right|
$$

Calculating the upper right derivative of $V_{1}(t)$ along system (1.4), we have

$$
\begin{aligned}
D^{+} V_{1}(t)= & \operatorname{sign}\left(x_{1}(t)-y_{1}(t)\right)\left[-a_{1}(t)\left(x_{1}(t)-y_{1}(t)\right)-f_{1}(t)\left(u_{1}(t)-v_{1}(t)\right)\right. \\
& -b_{1}(t)\left(\frac{x_{2}\left(t-\tau_{1}\right)}{c_{1}(t) x_{2}\left(t-\tau_{1}\right)+x_{1}\left(t-\tau_{1}\right)}-\frac{y_{2}\left(t-\tau_{1}\right)}{c_{1}(t) y_{2}\left(t-\tau_{1}\right)+y_{1}\left(t-\tau_{1}\right)}\right) \\
& \left.+d_{1}(t)\left(\frac{x_{3}\left(t-\tau_{1}\right)}{e_{1}(t) x_{3}\left(t-\tau_{1}\right)+x_{1}\left(t-\tau_{1}\right)}-\frac{y_{3}\left(t-\tau_{1}\right)}{e_{1}(t) y_{3}\left(t-\tau_{1}\right)+y_{1}\left(t-\tau_{1}\right)}\right)\right] \\
& +\operatorname{sign}\left(x_{2}(t)-y_{2}(t)\right)\left[-a_{2}(t)\left(x_{2}(t)-y_{2}(t)\right)+f_{2}(t)\left(u_{2}(t)-v_{2}(t)\right)\right. \\
& -b_{2}(t)\left(\frac{x_{1}\left(t-\tau_{2}\right)}{c_{2}(t) x_{1}\left(t-\tau_{2}\right)+x_{2}\left(t-\tau_{2}\right)}-\frac{y_{1}\left(t-\tau_{2}\right)}{c_{2}(t) y_{1}\left(t-\tau_{2}\right)+y_{2}\left(t-\tau_{2}\right)}\right) \\
& \left.+d_{2}(t)\left(\frac{x_{3}\left(t-\tau_{2}\right)}{e_{2}(t) x_{3}\left(t-\tau_{2}\right)+x_{2}\left(t-\tau_{2}\right)}-\frac{y_{3}\left(t-\tau_{2}\right)}{e_{2}(t) y_{3}\left(t-\tau_{2}\right)+y_{2}\left(t-\tau_{2}\right)}\right)\right] \\
& +\operatorname{sign}\left(x_{3}(t)-y_{3}(t)\right)\left[-a_{3}(t)\left(x_{3}(t)-y_{3}(t)\right)+f_{3}(t)\left(u_{3}(t)-v_{3}(t)\right)\right. \\
& +g_{1}(t)\left(\frac{x_{1}\left(t-\tau_{3}\right)}{e_{1}(t) x_{3}\left(t-\tau_{3}\right)+x_{1}\left(t-\tau_{3}\right)}-\frac{y_{1}\left(t-\tau_{3}\right)}{e_{1}(t) y_{3}\left(t-\tau_{3}\right)+y_{1}\left(t-\tau_{3}\right)}\right) \\
& +g_{2}(t)\left(\frac{x_{2}\left(t-\tau_{3}\right)}{e_{2}(t) x_{3}\left(t-\tau_{3}\right)+x_{2}\left(t-\tau_{3}\right)}-\frac{y_{2}\left(t-\tau_{3}\right)}{e_{2}(t) y_{3}\left(t-\tau_{3}\right)+y_{2}\left(t-\tau_{3}\right)}\right] \\
= & \operatorname{sign}\left(x_{1}(t)-y_{1}(t)\right)\left[-a_{1}(t)\left(x_{1}(t)-y_{1}(t)\right)-f_{1}(t)\left(u_{1}(t)-v_{1}(t)\right)\right.
\end{aligned}
$$




$$
\begin{aligned}
& -C_{2}(t)\left(x_{2}\left(t-\tau_{1}\right)-y_{2}\left(t-\tau_{1}\right)\right)+C_{4}(t)\left(x_{3}\left(t-\tau_{1}\right)-y_{3}\left(t-\tau_{1}\right)\right) \\
& \left.+\left(C_{1}(t)-C_{3}(t)\right)\left(x_{1}\left(t-\tau_{1}\right)-y_{1}\left(t-\tau_{1}\right)\right)\right]+\operatorname{sign}\left(x_{2}(t)-y_{2}(t)\right) \\
& \times\left[-a_{2}(t)\left(x_{2}(t)-y_{2}(t)\right)+f_{2}(t)\left(u_{2}(t)-v_{2}(t)\right)-C_{5}(t)\left(x_{1}\left(t-\tau_{2}\right)\right.\right. \\
& \left.-y_{1}\left(t-\tau_{2}\right)\right)+\left(C_{6}(t)-C_{7}(t)\right)\left(x_{2}\left(t-\tau_{2}\right)-y_{2}\left(t-\tau_{2}\right)\right)+C_{8}(t) \\
& \left.\times\left(x_{3}\left(t-\tau_{2}\right)-y_{3}\left(t-\tau_{2}\right)\right)\right]+\operatorname{sign}\left(x_{3}(t)-y_{3}(t)\right)\left[-a_{2}(t)\left(x_{2}(t)-y_{2}(t)\right)\right. \\
& +f_{3}(t)\left(u_{3}(t)-v_{3}(t)\right)-\left(C_{10}(t)+C_{12}(t)\right)\left(x_{3}\left(t-\tau_{3}\right)-y_{3}\left(t-\tau_{3}\right)\right) \\
& \left.+C_{9}(t)\left(x_{1}\left(t-\tau_{3}\right)-y_{1}\left(t-\tau_{3}\right)\right)+C_{11}(t)\left(x_{2}\left(t-\tau_{3}\right)-y_{2}\left(t-\tau_{3}\right)\right)\right] \\
\leq & -\sum_{i=3}^{3} a_{i}^{L}\left|x_{i}(t)-y_{i}(t)\right|+\sum_{i=3}^{3} f_{i}^{M}\left|u_{i}(t)-v_{i}(t)\right|+C_{2}^{M}\left|x_{2}\left(t-\tau_{1}\right)-y_{2}\left(t-\tau_{1}\right)\right| \\
& +\left(C_{1}^{M}+C_{3}^{M}\right)\left|x_{1}\left(t-\tau_{1}\right)-y_{1}\left(t-\tau_{1}\right)\right|+C_{4}^{M}\left|x_{3}\left(t-\tau_{1}\right)-y_{3}\left(t-\tau_{1}\right)\right| \\
& +\left(C_{6}^{M}+C_{7}^{M}\right)\left|x_{2}\left(t-\tau_{2}\right)-y_{2}\left(t-\tau_{2}\right)\right|+C_{5}^{M}\left|x_{1}\left(t-\tau_{2}\right)-y_{1}\left(t-\tau_{2}\right)\right| \\
& +C_{8}^{M}\left|x_{3}\left(t-\tau_{2}\right)-y_{3}\left(t-\tau_{2}\right)\right|+\left(C_{10}^{M}+C_{12}^{M}\right)\left|x_{3}\left(t-\tau_{3}\right)-y_{3}\left(t-\tau_{3}\right)\right| \\
& +C_{9}^{M}\left|x_{1}\left(t-\tau_{3}\right)-y_{1}\left(t-\tau_{3}\right)\right|+C_{11}^{M}\left|x_{2}\left(t-\tau_{3}\right)-y_{2}\left(t-\tau_{3}\right)\right| .
\end{aligned}
$$

Next, we let

$$
\begin{aligned}
V_{2}(t)= & \left(C_{1}^{M}+C_{3}^{M}\right) \int_{t-\tau_{1}}^{t}\left|x_{1}(s)-y_{1}(s)\right| d s+C_{2}^{M} \int_{t-\tau_{1}}^{t}\left|x_{2}(s)-y_{2}(s)\right| d s \\
& +C_{4}^{M} \int_{t-\tau_{1}}^{t}\left|x_{3}(s)-y_{3}(s)\right| d s+\left(C_{6}^{M}+C_{7}^{M}\right) \int_{t-\tau_{2}}^{t}\left|x_{2}(s)-y_{2}(s)\right| d s \\
& +C_{5}^{M} \int_{t-\tau_{2}}^{t}\left|x_{1}(s)-y_{1}(s)\right| d s+C_{8}^{M} \int_{t-\tau_{2}}^{t}\left|x_{3}(s)-y_{3}(s)\right| d s \\
& +\left(C_{10}^{M}+C_{12}^{M}\right) \int_{t-\tau_{3}}^{t}\left|x_{3}(s)-y_{3}(s)\right| d s+C_{9}^{M} \int_{t-\tau_{3}}^{t}\left|x_{1}(s)-y_{1}(s)\right| d s \\
& +C_{11}^{M} \int_{t-\tau_{3}}^{t}\left|x_{2}(s)-y_{2}(s)\right| d s .
\end{aligned}
$$

Calculating the upper right derivative of $V_{2}(t)$ and from (4.1), we have

$$
\begin{aligned}
D^{+} V_{1}(t)+D^{+} V_{2}(t) \leq & -\left(a_{1}^{L}-C_{1}^{M}-C_{3}^{M}-C_{5}^{M}-C_{9}^{M}\right)\left|x_{1}(t)-y_{1}(t)\right| \\
& -\left(a_{2}^{L}-C_{2}^{M}-C_{6}^{M}-C_{7}^{M}-C_{11}^{M}\right)\left|x_{2}(t)-y_{2}(t)\right| \\
& -\left(a_{3}^{L}-C_{4}^{M}-C_{8}^{M}-C_{10}^{M}-C_{12}^{M}\right)\left|x_{3}(t)-y_{3}(t)\right| \\
& +f_{1}^{M}\left|u_{1}(t)-v_{1}(t)\right|+f_{2}^{M}\left|u_{2}(t)-v_{2}(t)\right|+f_{3}^{M}\left|u_{3}(t)-v_{3}(t)\right| .
\end{aligned}
$$

Moreover, we let

$$
V_{3}(t)=\sum_{i=1}^{3}\left|u_{i}(t)-v_{i}(t)\right| .
$$


Calculating the upper right derivative of $V_{3}(t)$ and from (4.3), we have

$$
\begin{aligned}
\sum_{i=1}^{3} D^{+} V_{i}(t) \leq & -\left(a_{1}^{L}-C_{1}^{M}-C_{3}^{M}-C_{5}^{M}-C_{9}^{M}-h_{1}^{M}\right)\left|x_{1}(t)-y_{1}(t)\right| \\
& -\left(a_{2}^{L}-C_{2}^{M}-C_{6}^{M}-C_{7}^{M}-C_{11}^{M}-h_{2}^{M}\right)\left|x_{2}(t)-y_{2}(t)\right| \\
& -\left(a_{3}^{L}-C_{4}^{M}-C_{8}^{M}-C_{10}^{M}-C_{12}^{M}-h_{3}^{M}\right)\left|x_{3}(t)-y_{3}(t)\right| \\
& -\sum_{i=1}^{3}\left(p_{i}^{L}-f_{i}^{M}\right)\left|u_{i}(t)-v_{i}(t)\right| .
\end{aligned}
$$

Finally, we let a Lyapunov function be as follows:

$$
V(t)=V_{1}(t)+V_{2}(t)+V_{3}(t) .
$$

Calculating the upper right derivation of $V(t)$, from (4.4) we finally can obtain, for all $t \geq T$,

$$
D^{+} V(t) \leq-\sum_{i=1}^{3}\left(D_{i}\left|x_{i}(t)-y_{i}(t)\right|+E_{i}\left|u_{i}(t)-v_{i}(t)\right|\right)
$$

Integrating from $T$ to $t$ on both sides of (4.5) produces

$$
V(t)+G \int_{T_{4}}^{t}\left(\sum_{i=1}^{3}\left[\left|x_{i}(s)-y_{i}(s)\right|+\left|u_{i}(s)-v_{i}(s)\right|\right]\right) d s \leq V(T)<+\infty .
$$

Hence, $V(t)$ is bounded on $[T, \infty)$, and we have

$$
\int_{T_{4}}^{t}\left(\sum_{i=1}^{3}\left[\left|x_{i}(s)-y_{i}(s)\right|+\left|u_{i}(s)-v_{i}(s)\right|\right]\right) d s \leq \frac{V(T)}{G}<+\infty .
$$

Then we have

$$
\sum_{i=1}^{3}\left(\left|x_{i}(s)-y_{i}(s)\right|+\left|u_{i}(s)-v_{i}(s)\right|\right) \in L^{1}(T,+\infty)
$$

From the permanence of system (1.4), we can obtain that $\sum_{i=1}^{3}\left(\left|x_{i}(s)-y_{i}(s)\right|+\mid u_{i}(s)-\right.$ $\left.v_{i}(s) \mid\right)$ is uniformly continuous on $[T,+\infty)$. By Barbalat's lemma it follows that

$$
\lim _{t \rightarrow \infty}\left|x_{i}(t)-y_{i}(t)\right|=0, \quad \lim _{t \rightarrow \infty}\left|u_{i}(t)-v_{i}(t)\right|=0, \quad(i=1,2,3)
$$

This completes the proof of Theorem 4.1.

Corollary 4.1 Suppose that the conditions of Corollary 3.1 hold and $G>0$, then system (1.4) has a positive $\omega$-periodic solution which is globally attractive. 


\section{One example}

In this section one example is given to illustrate the effectiveness of our results obtained in this paper.

Example We consider the following system:

$$
\begin{aligned}
& \dot{x}_{1}(t)= x_{1}(t)\left[4.45+0.35 \cos (t)-(4.15+0.35 \cos (t)) x_{1}(t)\right. \\
&-\frac{(0.75+0.2 \cos (t)) x_{2}(t-0.75)}{(3+0.25 \cos (t)) x_{2}(t-0.75)+x_{1}(t-0.75)} \\
&+\frac{(2.6+0.25 \cos (t)) x_{3}(t-0.75)}{(2.25+0.25 \cos (t)) x_{3}(t-0.75)+x_{1}(t-0.75)} \\
&\left.-(0.25+0.15 \cos (t)) u_{1}(t)\right] \\
& \dot{x}_{2}(t)= x_{2}(t)\left[4.5+0.45 \cos (t)-(4.2+0.25 \cos (t)) x_{2}(t)\right. \\
&-\frac{(0.85+0.2 \cos (t)) x_{1}(t-0.5)}{(3+0.35 \cos (t)) x_{1}(t-0.5)+x_{2}(t-0.5)} \\
&\left.+\frac{(2.75+0.2 \cos (t)) x_{3}(t-0.5)}{(2.35+0.35 \cos (t)) x_{3}(t-0.5)+x_{2}(t-0.5)}+(0.2+0.15 \cos (t)) u_{2}(t)\right], \\
& \dot{x}_{3}(t)= x_{3}(t)\left[3.75+0.35 \cos (t)-(4.3+0.25 \cos (t)) x_{3}(t)\right. \\
&+\frac{(0.4+0.2 \cos (t)) x_{1}(t-0.25)}{(3+0.15 \cos (t)) x_{3}(t-0.25)+x_{1}(t-0.25)} \\
&\left.+\frac{(0.35+0.1 \cos (t)) x_{2}(t-0.25)}{(2.15+0.15 \cos (t)) x_{3}(t-0.25)+x_{2}(t-0.25)}+(0.2+0.1 \cos (t)) u_{3}(t)\right], \\
& \dot{u}_{1}(t)= 2.25+0.35 \cos (t)-(1.95+0.25 \cos (t)) u_{1}(t)+(0.2+0.1 \cos (t)) x_{1}(t), \\
& \dot{u}_{2}(t)=2.65+0.25 \cos (t)-(1.85+0.35 \cos (t)) u_{2}(t)-(0.2+0.15 \cos (t)) x_{2}(t), \\
& \dot{u}_{2}(t)=1.65+0.15 \cos (t)-(1.75+0.45 \cos (t)) u_{3}(t)-(0.15+0.12 \cos (t)) x_{3}(t) .
\end{aligned}
$$

By direct calculation, we can get

$$
\begin{aligned}
& M_{1} \approx 1.6382, \quad M_{2} \approx 1.7979, \quad M_{3} \approx 1.3742, \quad m_{1} \approx 0.7323, \\
& m_{2} \approx 0.8959, \quad m_{3} \approx 0.7473, \quad N_{1} \approx 1.8185, \quad N_{2} \approx 1.9333 \text {, } \\
& N_{3} \approx 1.3846, \quad n_{1} \approx 0.8636, \quad n_{2} \approx 0.8049, \quad n_{3} \approx 0.5132, \\
& \gamma_{1}^{L} \approx 10.2143, \quad \gamma_{2}^{L} \approx 4.9587, \quad \gamma_{3}^{L} \approx 8.0458, \quad \gamma_{4}^{L} \approx 5.7139, \\
& C_{1}^{M} \approx 0.1672, \quad C_{2}^{M} \approx 0.1524, \quad C_{3}^{M} \approx 0.7898, \quad C_{4}^{M} \approx 0.9415, \\
& C_{5}^{M} \approx 0.2346, \quad C_{6}^{M} \approx 0.2138, \quad C_{7}^{M} \approx 0.7095, \quad C_{8}^{M} \approx 0.9282, \\
& C_{9}^{M} \approx 0.4157, \quad C_{10}^{M} \approx 0.4955, \quad C_{11}^{M} \approx 0.2922, \quad C_{12}^{M} \approx 0.3823, \\
& D_{1} \approx 1.8927 \quad D_{2} \approx 2.2322, \quad D_{3} \approx 1.0324, \quad E_{1} \approx 1.3, \quad E_{2} \approx 1.15 \text {, } \\
& E_{3} \approx 1, \quad G=1 .
\end{aligned}
$$



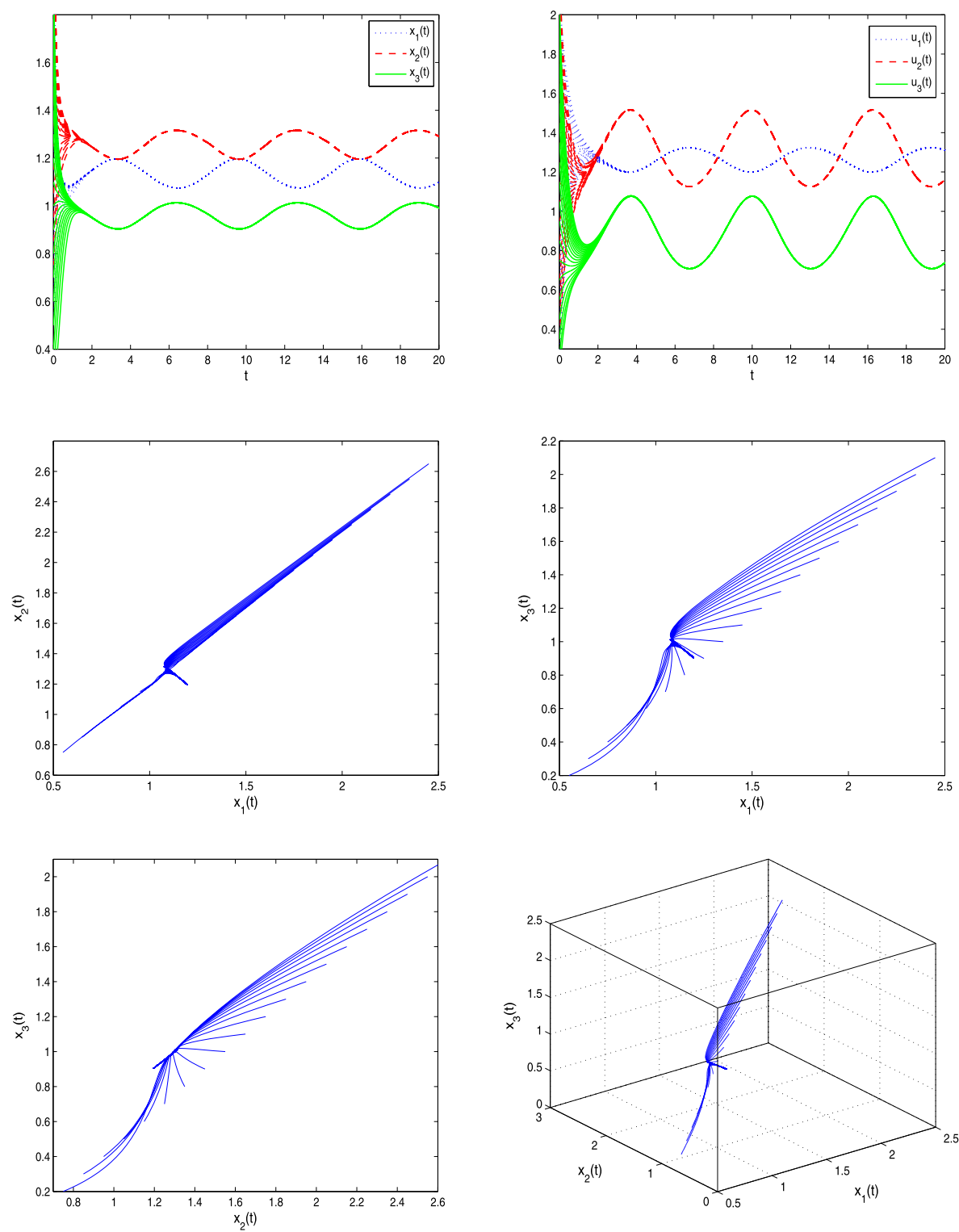

Figure 1 The numerical solutions of system (5.1) with different initial conditions

It is easy to show that system (5.1) satisfies the conditions of Theorem 3.2, Theorem 4.1, Corollary 3.1, and Corollary 4.1. Hence, system (5.1) is permanent, globally attractive and has a globally attractive positive periodic solution.

From Fig. 1 we can see that system (5.1) is permanent and has a globally attractive positive periodic solution.

\section{Conclusion}

In this study, we are concerned with system (1.4). First, using the inequality techniques and the comparison method, we obtained a set of conditions that ensure that the system is permanent and at least has a positive periodic solution. Second, using the Lyapunov function method, we derived sufficient conditions on the global attractivity of the system. 
Finally, we provided a suitable example to illustrate the feasibility of our main results. Because we extended systems (1.2) and (1.3) to system (1.4), we also obtained some sufficient conditions for the permanence, periodic solution, and global attractivity of system (1.4). Hence, system (1.4) and the results obtained in this study can be seen as the supplements and extensions of previously known studies $[13,17,20]$.

\section{Acknowledgements \\ Not applicable.}

\section{Funding}

This project is supported by the National Natural Science Foundation of Xinjiang (Grant No. 2021D01C067), the National Natural Science Foundation of China (Grant No. 11662020, 11861063) and the Tianshan Youth Program-Training Program for Excellent Young Scientific and Technological Talents (Grant No. 2019Q017).

\section{Availability of data and materials}

Not applicable.

\section{Competing interests}

The authors declare that they have no competing interests.

Authors' contributions

All authors contributed equally to this work. All authors read and approved the final manuscript.

\section{Publisher's Note}

Springer Nature remains neutral with regard to jurisdictional claims in published maps and institutional affiliations.

Received: 23 December 2020 Accepted: 3 April 2021 Published online: 01 May 2021

\section{References}

1. Zhang, Z:: Mutualism or cooperation among competitors promotes coexistence and competitive ability. Ecol. Model. $164,271-282(2003)$

2. Lotka, A.J.: Elements of Physical Biology. Williams \& Wilkins, Baltimore (1925)

3. Volterra, V.: Fluctuations in the abundance of a species considered mathematically. Nature 118, 558-560 (1926)

4. Ahmad, S.: On the nonautonomous Volterra-Lotka competition equations. Proc. Am. Math. Soc. 117, 199-204 (1993)

5. Fan, M., Wang, K., Jiang, D.: Existence and global attractivity of positive periodic solutions of periodic n-species Lotka-Volterra competition systems with several deviating arguments. Math. Biosci. 160, 47-61 (1999)

6. Chen, F:: The permanence and global attractivity of Lotka-Volterra competition system with feedback controls. Nonlinear Anal., Real World Appl. 7, 133-143 (2006)

7. Cui, J.A.: Global asymptotic stability in n-species cooperative system with time delays. Syst. Sci. Math. Sci. 7, 45-48 (1994)

8. Liu, Z., Wu, J., Tan, R., Chen, Y.: Modeling and analysis of a periodic delayed two-species model of facultative mutualism. Appl. Math. Comput. 217, 893-903 (2010)

9. Muhammadhaji, A., Teng, Z.: Global attractivity of a periodic delayed n-species model of facultative mutualism. Discrete Dyn. Nat. Soc. 2013,1-11 (2013)

10. Hsu, S., Hwang, T., Kuang, Y.: Rich dynamics of a ratio-dependent one prey two predators model. J. Math. Biol. 43, 377-396 (2001)

11. Li, H., Zhang, L., Hu, C., Jiang, Y., Teng, Z.: Dynamical analysis of a fractional-order predator-prey model incorporating a prey refuge. J. Appl. Math. Comput. 54, 435-449 (2017)

12. Muhammadhaji, A., Teng, Z., Zhang, L.: Permanence in general nonautonomous predator-prey Lotka-Volterra systems with distributed delays and impulses. J. Biol. Syst. 21, 1-28 (2013)

13. Wang, C., Li, L., Zhou, Y., Li, R.: On a delay ratio-dependent predator-prey system with feedback controls and shelter for the prey. Int. J. Biomath. 11(7), Article ID 1850095 (2018). https://doi.org/10.1142/S179352451850095X

14. Wang, C., Li, N., Zhou, Y., et al.: On a multi-delay Lotka-Volterra predator-prey model with feedback controls and prey diffusion. Acta Math. Sci. 39(2), 429-448 (2019)

15. Wang, C., Jia, L., Li, L., Wei, W.: Global stability in a delayed ratio-dependent predator-prey system with feedback controls. IAENG Int. J. Appl. Math. 50(3), 690-698 (2020)

16. Jia, L.: Analysis for a delayed three-species predator-prey model with feedback controls and prey diffusion. J. Math. 2020, Article ID 5703859 (2020). https://doi.org/10.1155/2020/5703859

17. Jia, L., Wang, C.: Persistence of a Lotka-Volterra ratio-dependent predator-prey model with delays and feedback controls. IOP Conf. Ser., Mater. Sci. Eng. 790, 012129 (2020). https://doi.org/10.1088/1757-899X/790/1/012129

18. Muhammadhaji, A., Teng, Z., Rahim, M.: Dynamical behavior for a class of delayed competitive-mutualism systems. Differ. Equ. Dyn. Syst. 23, 281-301 (2015)

19. Wang, Y., Wu, H.: A mutualism-competition model characterizing competitors with mutualism at low density. Math. Comput. Model. 53, 1654-1663 (2011)

20. Gyllenberg, M., Yan, P., Wang, Y.: Limit cycles for the competitor-competitor-mutualist Lotka-Volterra systems. Phys. D, Nonlinear Phenom. 221, 135-145 (2006) 
21. LV, X., Yan, P., Lu, S.: Existence and global attractivity of positive periodic solution of competitor-competitor-mutualist Lotka-Volterra system with deviating arguments. Math. Comput. Model. 51, 823-832 (2010)

22. Wang, C., Zhou, Y., Li, Y., Li, R.: Well-posedness of a ratio-dependent Lotka-Volterra system with feedback control. Bound. Value Probl. 2018, 117 (2018)

23. Yang, L., Xie, X., Chen, F., Xue, Y.: Permanence of the periodic predator-prey-mutualist system. Adv. Differ. Equ. 2015, Article ID 331 (2015). https://doi.org/10.1186/s13662-015-0654-9

24. Luo, Y., Zhang, L., Teng, Z., De Angelis, D.L.: A parasitism-mutualism-predation model consisting of crows, cuckoos and cats with stage-structure and maturation delays on crows and cuckoos. J. Theor. Biol. 446, 212-228 (2018)

25. Wang, C., Li, L.R., Zhang, Q., Li, R.: Dynamical behaviour of a Lotka-Volterra competitive-competitive-cooperative model with feedback controls and time delays. J. Biol. Dyn. 13,43-68 (2019)

26. Muhammadhaji, A., Teng, Z.: Permanence and extinction analysis for a periodic competing predator-prey system with stage structure. Int. J. Dyn. Control 5, 858-871 (2017)

\section{Submit your manuscript to a SpringerOpen ${ }^{\circ}$ journal and benefit from:}

- Convenient online submission

Rigorous peer review

Open access: articles freely available online

- High visibility within the field

- Retaining the copyright to your article

Submit your next manuscript at $\gg$ springeropen.com 